\title{
BMJ Open Protocol for a scoping review of the qualitative literature on Indigenous infant feeding experiences
}

\author{
Hiliary Monteith (D , ${ }^{1}$ Tracey Galloway, ${ }^{2}$ Anthony J Hanley ${ }^{1}$
}

To cite: Monteith H, Galloway T, Hanley AJ. Protocol for a scoping review of the qualitative literature on Indigenous infant feeding experiences. BMJ Open 2021;11:e043476. doi:10.1136/ bmjopen-2020-043476

\section{- Prepublication history and} additional materials for this paper is available online. To view these files, please visit the journal online (http://dx.doi. org/10.1136/bmjopen-2020043476).

Received 04 August 2020 Revised 07 January 2021 Accepted 13 January 2021

Check for updates

(c) Author(s) (or their employer(s)) 2021. Re-use permitted under CC BY-NC. No commercial re-use. See rights and permissions. Published by BMJ.

${ }^{1}$ Nutritional Sciences, University of Toronto, Toronto, Ontario, Canada

${ }^{2}$ Anthropology, University of Toronto, Mississauga, Ontario, Canada

Correspondence to

Dr Anthony J Hanley; anthony.hanley@utoronto.ca

\section{ABSTRACT}

Introduction Prudent infant nutrition, including exclusive breastfeeding to 6 months, is essential for optimal shortterm and long-term health. Quantitative research to date has documented that many Indigenous communities have lower breastfeeding rates than the general population and that this gap in breastfeeding initiation and maintenance may have an important impact on chronic disease risk later in life. However, there are critical knowledge gaps in the literature regarding factors that influence infant feeding decisions. Qualitative research on infant feeding experiences provides a broader understanding of the challenges that Indigenous caregivers encounter, and insights provided by this approach are essential to identify research gaps, community engagement strategies, and programme and policy development. The objective of this review is to summarise the qualitative literature that describes breastfeeding and other infant feeding experiences of Indigenous caregivers.

Methods and analysis This scoping review will follow guidelines from Preferred Reporting Items for Systematic Reviews and Meta-Analyses Extension for Scoping Reviews, the Joanna Briggs Institute and the methodological framework from Arksey and O'Malley. In October 2020, we will conduct an electronic database search using Medline, Embase, The Cumulative Index to Nursing \& Allied Health Literature (CINAHL), PsycINFO, and Scopus, and will focus on qualitative studies. Publications that have a focus on infant feeding in Canada, the USA, Australia and New Zealand, and the Indigenous caregiver experience from the caregiver perspective, will be included. We will conduct a grey literature search using Indigenous Studies Portal, country-specific browser searches, and known government, association, and community websites/reports. We will map themes and concepts of the publications, including study results and methodologies, to identify research gaps, future directions, challenges and best practices in this topic area.

Ethics and dissemination Ethical approval is not required for this review as no unpublished primary data will be included. The results of this review will be shared through peer-reviewed publications and conference presentations. This protocol is registered through the Open Science Framework (osf.io/4su79).

\section{INTRODUCTION}

Indigenous peoples living in Canada, the USA, Australia and New Zealand are

\section{Strengths and limitations of this study}

- This protocol describes a rigorous search strategy and methodological framework for summarising the literature that align with the research question and include peer-reviewed sources, as well as a grey literature search.

- Selection of publications that meet the inclusion and exclusion criteria will be completed by two independent reviewers and most inclusion/exclusion criteria are only applied at screening, not at the search, augmenting the comprehensiveness of this review.

- This review will map important findings and methodologies to provide an overview of work in this area, guiding best practices for future projects.

- The topic of this review is broad and interdisciplinary; therefore, it is possible that publications only available in subject-specific databases or websites may be omitted.

disproportionately affected by chronic diseases, including type 2 diabetes mellitus. Their heavy disease burden is compounded by socioecological factors, such as food insecurity, poverty, housing and water sanitation issues. ${ }^{12}$ These adverse environments and funding limitations are a direct result of the legacy of colonisation and they restrict the ease in which Indigenous communities can improve their health and well-being. ${ }^{3-5}$ In recent years, there has been an emphasis in research inquiry and public health programming on the contribution of these complex interconnected factors to health disparities, and the increased recognition for the need for multidimensional and culturally safe approaches to support Indigenous communities into the future. ${ }^{4} 6$ The health and wellbeing of Indigenous infants and children are priorities for improved health outcomes overall, as maternal and early life risk factors are known to have long-term effects on health later in life. ${ }^{78}$ Importantly, a focus on infants and children also aligns with Indigenous ways of knowing, ${ }^{9}$ where intergenerational relationships hold particular significance. 
It has been well documented that Indigenous infants and children disproportionately experience risk factors that are associated with chronic diseases later in life, including high rates of overweight and obesity, food insecurity, poverty and limited quality of education. ${ }^{10-12}$ Optimal nutrition during infancy and childhood is an important factor that contributes broadly to health and well-being across the lifespan. ${ }^{13}{ }^{14}$ A limited number of previous studies of Indigenous infants have reported that breastfeeding initiation and duration have important protective effects on subsequent risk for type 2 diabetes and adiposity ${ }^{71516}$; however, breastfeeding rates are often low among Indigenous mothers in developed countries. ${ }^{17-19}$ Although quantitative studies have reported descriptive statistics and basic epidemiological features of breastfeeding among Indigenous mothers, many important knowledge gaps remain. Qualitative research decontextualises and recontextualises the deeper meanings and reasons for infant feeding experiences as perceived by Indigenous caregivers, providing clarity of the phenomena of interest, uncovering new understanding and possibly illuminating areas for further inquiry. ${ }^{20}$ Infant feeding initiatives must be informed by these experiences to better address community-specific concerns to effectively promote healthy infant feeding behaviours, including breastfeeding, within Indigenous communities. $^{21-24}$

\section{Rationale}

To date, there are no published summaries, scoping or systematic reviews on infant feeding experiences among Indigenous caregivers that include qualitative descriptions of barriers, stories, supports and initiatives or related topics. A scoping review in this area will assist researchers in understanding the current state of the existing literature, research gaps and key research priorities for future work. This review will also summarise the qualitative research methodologies used in Indigenous infant feeding studies with the potential to clarify best research practices, and additional methodological applications to address gaps in the literature. This information may also result in further clarification of clinical best practices for breast and alternative forms of infant feeding among Indigenous populations.

\section{Objectives}

The primary aim of this work is to summarise the literature available to date that incorporates qualitative approaches to describe the breastfeeding and other infant feeding experiences of Indigenous women residing in developed nations impacted by colonisation. This review will include research addressing Indigenous women's experiences from their own perspectives, as well as the perspectives of other caregivers, including but not limited to grandmothers and fathers. In addition to literature on breastfeeding experiences, this review will also include literature on alternative infant feeding options, including formula feeding, complementary feeding, weaning and
Table 1 Eligibility criteria overview

\begin{tabular}{|c|c|}
\hline Inclusion criteria & Exclusion criteria \\
\hline $\begin{array}{l}\text { Indigenous populations in } \\
\text { Canada, US, New Zealand } \\
\text { and Australia }\end{array}$ & $\begin{array}{l}\text { Work not describing } \\
\text { experiences from a } \\
\text { caregiver's perspective }\end{array}$ \\
\hline $\begin{array}{l}\text { Explores breastfeeding and } \\
\text { alternative infant feeding } \\
\text { options }\end{array}$ & $\begin{array}{l}\text { Work only about the } \\
\text { introduction to solid foods }\end{array}$ \\
\hline Published in English & No English version \\
\hline Published after 1969 & Published before 1969 \\
\hline $\begin{array}{l}\text { Qualitative or mixed methods } \\
\text { data }\end{array}$ & $\begin{array}{l}\text { Presentation of only } \\
\text { quantitative and numerical } \\
\text { data that do not describe } \\
\text { infant feeding experiences }\end{array}$ \\
\hline
\end{tabular}

other forms of milk feeding if the work describes the alternative in relation to breastfeeding.

\section{METHODS}

\section{Protocol and registration}

The protocol for this scoping review follows Preferred Reporting Items for Systematic Reviews and MetaAnalyses guidelines adapted for scoping reviews, ${ }^{25}$ as well as guidelines from the Joanna Briggs Institute Reviewer's Manua $^{26}$ and guidelines published in 2005 by Arksey and O'Malley. ${ }^{27}$ The protocol is registered with the Open Science Framework.

\section{Eligibility criteria}

Table 1 provides an overview of the inclusion and exclusion criteria for this scoping review. The population of focus is Indigenous peoples living in Canada, the USA, New Zealand and Australia. These countries are included as they are developed nations that have similar legacies of colonisation, where western worldview is dominant, and in which Indigenous peoples have similar health outcomes. ${ }^{28}{ }^{29}$ Infant feeding experiences are the main focus for this review. Breastfeeding, as well as alternative forms of infant feeding, such as formula and cow's milk, are included; however, we will exclude works that only focus on the introduction of solid foods. Breastfeeding compared with not breastfeeding within the same population is the comparison considered in this review, when applicable, where we consider caregiver experiences of breastfeeding compared with other infant feeding strategies. The qualitative outcomes specific to experiences, perspectives and practices (including themes, descriptions, open-ended survey responses or any answers pertaining to experience) as described from the caregiver or others involved in caregiving will be included; work that only describes an outsider perspective will be excluded.

Additional inclusion criteria include: works published in the English language, grey literature and peer-reviewed journal articles, work with a focus on Indigenous groups 
within Australia, Canada, New Zealand and the USA as the primary population, research using qualitative or mixed methods where the infant feeding experience is described, and works published after 1969. Works published prior to 1969 are likely to include literature that has been archived and is therefore not feasible to review in detail for this review. Should there be works that do not clearly fit within these criteria, the two reviewers will meet to discuss until consensus is reached. We will report where clarity was and was not achieved and disclose why. Publications that do not involve Indigenous populations will also be excluded.

\section{Information sources}

Databases included in the initial search for this review will be Medline, Embase, CINAHL, PsycINFO, and Scopus. These databases are selected for this review to include a broad range of research as our topic overlaps with various fields, including Anthropology, Health Sciences, Sociology and Indigenous Studies. Following this initial search, the grey literature will be explored for additional relevant documents.

The grey literature search will concentrate on resources and publications available from Indigenous Studies Portal and a variety of Indigenous focused websites, governments, organisations and book chapters. A thorough Google search will be conducted with each of the countryspecific Google versions (eg, Google AU) and the first 10 pages of results will be included in the search. Indigenous scholars and non-Indigenous scholars who work in this area of study in Canada, Australia, USA and New Zealand will be contacted with the aim of including as many applicable grey literature sources as needed to be as sensitive in our search as possible. The Canadian Agency for Drugs and Technologies in Health's 'Grey Matters' checklist ${ }^{30}$ will also be consulted.

Given the limitations to reproducibility and comprehensiveness in a grey literature search, transparency is particularly important. Therefore, the reporting strategy used for the grey literature search will include all websites (URL and title) visited, the dates of searches, the search terms used to reach such websites and used within those websites, and the number of items screened. We will report both the sources of relevant content, as well as when no relevant content is found on a website or from a specific search. ${ }^{26}$ We will also search book chapters and conference proceedings in the following databases: Medline, Embase, CINAHL, PsycINFO, Scopus and Indigenous Studies Portal. The initial database search and exporting of abstracts and references will take place from 20 to 27 October 2020.

The detailed proposed search strategy can be found as online supplemental appendix 1.

\section{Selection of sources of evidence}

All literature references will be exported to Zotero software (Corporation for Digital Scholarship, Virginia) and saved. The titles, abstracts and references will then be transferred to Covidence, ${ }^{31}$ where duplicates will be removed and data will be managed for the duration of the scoping review. This will enable independent review of the literature for the reviewers/authors.

A minimum of two independent reviewers will be involved in this work from screening to inclusion. At minimum, an additional author will assist in summarising the included literature and in writing the scoping review.

\section{Data charting process}

All data will be collected and shared using Covidence software $^{31}$ to enable an independent review process. The software facilitates the review process through organised management of the sources, and identification of publications where reviewers are not concordant and discussion may be required. If or when review decisions differ, an author other than the primary two reviewers will provide a third vote to achieve a review decision. All titles and abstracts will be screened by two reviewers at the screening stage and all eligible sources will move to a fulltext review, also completed by two independent reviewers.

\section{Data items}

Literature included in this scoping review must be from qualitative or mixed-method studies. Articles that report on survey data will be included if the questions reported on are based on infant feeding experience (perspectives, perceptions and practices), whether the survey questions were open ended or not. Data are considered as any information, such as quotations, codes, themes and openended survey responses as first, second and/or third order constructs, describing infant feeding experiences.

Experience is defined as 'practical knowledge, skill, or practice derived from direct observation of or participation in events or in a particular activity (Merriam-Webster. $\left.\mathrm{com}^{32}\right)^{\prime}$. In this work, experience refers to the reported knowledge, skill or practice from direct observation or participation in infant feeding.

The term "Indigenous peoples" has not officially been defined by the United Nations given the importance of enabling Indigenous peoples to self-determine their identity and that a specific definition is not required for the protection of Indigenous rights. ${ }^{3}$ A working definition is provided by the José R. Martínez Cobo Study and is as follows:

Indigenous communities, peoples and nations are those which, having a historical continuity with preinvasion and pre-colonial societies that developed on their territories, consider themselves distinct from other sectors of the societies now prevailing on those territories, or parts of them. They form at present non-dominant sectors of society and are determined to preserve, develop and transmit to future generations their ancestral territories, and their ethnic identity, as the basis of their continued existence as peoples, in accordance with their own cultural patterns, social institutions and legal system. ${ }^{33}$ 
In Canada, Indigenous groups include Inuit, Métis, and First Nations, including any of the over 600 recognised First Nations. In Australia, this includes Aboriginal and Torres Straight Islanders, in New Zealand, the Maori people, in the USA, Native American peoples and Alaska Natives. $^{3}$

Breastfeeding is a form of infant/early childhood nutrition using breast milk. In this scoping review, breastfeeding as well as any other form of infant feeding such as formula feeding, cow's milk administration, bottle feeding, expressed milk feeding, milk bank feeding, wet nurse feeding and others are included so long as the work describes the breastfeeding or absence of breastfeeding experience. For those works that only describe an alternative method to breastfeeding, the work must describe that method in relation to breastfeeding (ie, Why breastfeeding was not engaged etc).

\section{Synthesis of results}

As previously mentioned, Covidence software ${ }^{31}$ will be used to manage the literature and selection process. Once the records have been screened and full-text articles have been reviewed, studies that meet the inclusion criteria will be retained. This literature will be synthesised based on charting of results and thematic analysis. This process of synthesising the results will be completed by the primary author with the feedback and review of the second and third authors. The results will focus on the themes, quotations, conclusions and other interpretations related to infant feeding experiences of Indigenous caregivers, as well as a synopsis of the methodologies and theories used to support the work. Two steps will be used to present the findings: (1) a figure highlighting the number of studies at each stage of the search and (2) a written analysis of the primary outcomes. Qualitative evidence synthesis can have several challenges; therefore, detailed documentation will be important for the analytical process, including decision-making rationale through mind mapping and/or charting. ${ }^{34}$ The final results will be validated by a researcher in the field and an Indigenous community member with lived experience.

Validation of sources will be conducted using a test set of preidentified relevant publications that are expected to be captured using the database search terms. After the first database search is complete, we will check to see if these publications are included in our strategy. If they are, this will indicate that our search was likely comprehensive, if they are not included, we will investigate why, report this information, and make the appropriate changes to the search strategy prior to searching the remaining databases.

This paper describes the protocol for a scoping review of peer reviewed journal articles and grey literature on the topic of qualitative research on infant feeding experiences of Indigenous caregivers living in Canada, Australia, the USA and New Zealand. There is a need to explore and understand the literature related to Indigenous people's experiences with infant feeding practices as no such review exists to date. This is an important knowledge gap given the significant role that infant feeding plays in Indigenous health and well-being, and disease prevention. This scoping review will summarise the literature to date and highlight any important gaps that exist to guide research priorities in the future. It is anticipated that this review will also summarise the methodologies used to date, providing guidance for future research, highlighting best practices and/or gaps in how data have been collected.

Acknowledgements The authors would like to thank our Research Librarian, Glyneva Bradley-Ridout, at the University of Toronto, for her guidance in the scoping review process.

Contributors HM contributed to the drafting and editing of the protocol and oversaw revisions. TG provided feedback on the structure of the manuscript and the search strategy. AH contributed to the introduction and methods and was extensively involved in editing the manuscript. All authors approved the final manuscript.

Funding This research received no specific grant from any funding agency in the public, commercial or not-for-profit sectors. TG is supported by the Canadian Institutes of Health Research, Fund Number 147826. HM is supported by an Ontario Graduate Scholarship and the following University of Toronto (U of T) scholarships: a Banting and Best Diabetes Centre Scholarship, a Department of Nutritional Sciences Loblaw Food as Medicine Award, the Dr. Bernard Lau Memorial Scholarship (B): Graduate Bursary, the Al and Hannah Perly Graduate Student Scholarship, and the Peterborough K.M. Hunter Graduate Scholarship.

\section{Competing interests None declared.}

Patient and public involvement statement Patients or the public were not involved in the design, or conduct, or reporting, or dissemination plans of this research.

\section{Patient consent for publication Not required.}

Ethics approval As this review involves material previously published or in the public domain, ethical approval is not required; however, this review is specific to Indigenous groups and therefore, it is important to consider data sovereignty and ethics in the analysis and interpretation of results. The methodologies of the included works will be considered within this context, and the reviewers will validate results with an Indigenous scholar and/or community member prior to publication.

Provenance and peer review Not commissioned; externally peer reviewed.

Supplemental material This content has been supplied by the author(s). It has not been vetted by BMJ Publishing Group Limited (BMJ) and may not have been peer-reviewed. Any opinions or recommendations discussed are solely those of the author(s) and are not endorsed by BMJ. BMJ disclaims all liability and responsibility arising from any reliance placed on the content. Where the content includes any translated material, BMJ does not warrant the accuracy and reliability of the translations (including but not limited to local regulations, clinical guidelines, terminology, drug names and drug dosages), and is not responsible for any error and/or omissions arising from translation and adaptation or otherwise.

Open access This is an open access article distributed in accordance with the Creative Commons Attribution Non Commercial (CC BY-NC 4.0) license, which permits others to distribute, remix, adapt, build upon this work non-commercially, and license their derivative works on different terms, provided the original work is properly cited, appropriate credit is given, any changes made indicated, and the use is non-commercial. See: http://creativecommons.org/licenses/by-nc/4.0/.

\section{ORCID iD}

Hiliary Monteith http://orcid.org/0000-0002-7123-5910

\section{REFERENCES}

1 United Nations, Department of Economic and Social Affairs. Indigenous Peoples: Health [online]. Available: https://www.un.org/ development/desa/indigenouspeoples/mandated-areas1/health.html 
2 Knibbs LD, Sly PD. Indigenous health and environmental risk factors: an Australian problem with global analogues? Glob Health Action 2014;7:23766.

3 United Nations. State of the worlds indigenous peoples: indigenous peoples' access to health services [online], 2016. Available: https:// www.un.org/esa/socdev/unpfii/documents/2016/Docs-updates/ SOWIP_Health.pdf

4 Asia Pacific Forum, United Nations. The united nations declaration on the rights of indigenous peoples: a manual for national human rights institutions [online], 2013. Available: https://www.ohchr.org/ documents/issues/ipeoples/undripmanualfornhris.pdf

5 Sheikh MA, Islam R. Cultural and socio-economic factors in health, health services and prevention for Indigenous people. Antrocom Online J Anthropol 2010;6:263-73.

6 Dalang R, Carino J. Indigenous peoples and the human rightsbased approach to development: engaging in dialogue [online]. United Nations Development Programme (UNDP), 2007. Available: https://www.undp.org/content/dam/rbap/docs/Research\%20\&\% 20Publications/democratic_governance/RBAP-DG-2007-IndigenousPeoples-Approach-to-Development.pdf

7 McNamara BJ, Gubhaju L, Chamberlain C, et al. Early life influences on cardio-metabolic disease risk in aboriginal populations--what is the evidence? A systematic review of longitudinal and case-contro studies. Int J Epidemiol 2012;41:1661-82.

8 Martens PJ, Shafer LA, Dean HJ, et al. Breastfeeding initiation associated with reduced incidence of diabetes in mothers and offspring. Obstet Gynecol 2016;128:1095-104.

9 Greenwood M. Children as citizens of first nations: linking Indigenous health to early childhood development. Paediatr Child Health 2005;10:553-5.

10 First Nations Information Governance Centre. National report of the first nations regional health survey phase 3: volume two. first nations information governance centre 2018.

11 Government of Canada SC. Dietary habits of aboriginal children [online], 2013. Available: https://www150.statcan.gc.ca/n1/pub/82003-x/2013004/article/11776-eng.htm

12 Willows ND, Johnson MS, Ball GDC. Prevalence estimates of overweight and obesity in Cree preschool children in northern Quebec according to international and US reference criteria. Am J Public Health 2007;97:311-6.

13 Ip S, Chung M, Raman G, et al. Breastfeeding and maternal and infant health outcomes in developed countries. Evid Rep Technol Assess 2007;153:1-186.

14 Dieterich CM, Felice JP, O'Sullivan E, et al. Breastfeeding and health outcomes for the mother-infant dyad. Pediatr Clin North Am 2013;60:31-48.

15 Pettitt DJ, Forman MR, Hanson RL, et al. Breastfeeding and incidence of non-insulin-dependent diabetes mellitus in Pima Indians. Lancet 1997;350:166-8.

16 Willows ND, Morel J, Gray-Donald K. Prevalence of anemia among James Bay Cree infants of northern Quebec. CMAJ 2000;162:323-6.

17 Kuperberg K, Evers S. Feeding patterns and weight among first nations children. Can J Diet Pract Res 2006;67:79-84.
18 Chamberlain CR, Wilson AN, Amir LH, et al. Low rates of predominant breastfeeding in hospital after gestational diabetes, particularly among Indigenous women in Australia. Aust N Z J Public Health 2017;41:144-50.

19 Mclsaac KE, Lou W, Sellen D, et al. Exclusive breastfeeding among Canadian Inuit: results from the Nunavut Inuit child health survey. $J$ Hum Lact 2014;30:229-41.

20 Becker H. Chapter 4: Concepts. In: Tricks of the trade: how to think about your research while you're doing it. University of Chicago Press, 1998: 109-45.

21 Dodgson J, Struthers R. Traditional breastfeeding practices of the Ojibwe of northern Minnesota. Health Care Women Int 2003;24:49-61.

22 Eni R, Phillips-Beck W, Mehta P. At the edges of embodiment: determinants of breastfeeding for first nations women. Breastfeed Med 2014;9:203-14.

23 Moffitt P, Dickinson R. Creating exclusive breastfeeding knowledge translation tools with first nations mothers in northwest territories, Canada. Int J Circumpolar Health 2016;75:32989.

24 Houghtaling B, Byker Shanks C, Ahmed S, et al. Grandmother and health care professional breastfeeding perspectives provide opportunities for health promotion in an American Indian community. Soc Sci Med 2018;208:80-8.

25 Tricco AC, Lillie E, Zarin W, et al. PRISMA extension for scoping reviews (PRISMA-ScR): checklist and explanation. Ann Intern Med 2018;169:467-73.

26 Peters M, Godfrey C, Mclnerney P. Chapter 11: Scoping reviews (2020 Version). In: Aromataris E, Munn Z, eds. Joanna Briggs Institute reviewer's manual [online]. JBI, 2020. https://reviewersmanual. joannabriggs.org/

27 Arksey H, O'Malley L. Scoping studies: towards a methodological framework. Int J Soc Res Methodol 2005;8:19-32.

28 Smylie J, Crengle S, Freemantle J. Indigenous birth outcomes in Australia, Canada, New Zealand and the United States - an overview. Open Womens Health J 2010;4:7-17.

29 Yeates KE, Cass A, Sequist TD, et al. Indigenous people in Australia, Canada, New Zealand and the United States are less likely to receive renal transplantation. Kidney Int 2009;76:659-64.

30 Canadian Agency for Drugs and Technologies in Health (CADTH) Grey matters: a practical tool for searching health-related grey literature, 2015.

31 Covidence systematic review software [online]. Melbourne AU: Veritas Health Innovation. Available: www.covidence.org

32 Merriam-Webster. Experience [online], 2020. Available: https://www. merriam-webster.com/dictionary/experience

33 Martinez Cobo J. Problem of discrimination against Indigenous populations, E/CN.4/Sub.2/1986/7/Add.4, para. 379. United nations, 1986.

34 Soilemezi D, Linceviciute S. Synthesizing qualitative research: reflections and lessons learnt by two new reviewers. Int J Qual Methods 2018;17. 